Satellite Symposium

\title{
The Low Income Diet and Nutrition Survey: overview of methods
}

\author{
M. Bush \\ Food Standards Agency, 125 Kingsway, London WC2B 6NH, UK
}

The Low Income Diet and Nutrition Survey (LIDNS) was carried out to secure robust information on food consumption, nutrient intakes and nutritional status and the factors affecting these variables in materially-deprived households.

The survey successfully secured a sample that was representative of the $15 \%$ most-deprived households in the UK using a multi-stage clustered design to select potential participants. Eligibility for inclusion was established 'on the doorstep' based on a range of indicators and the household's net income.

Key stages in the survey were: an extensive face-to-face computer-assisted personal interview. This interview covered: factors that could influence dietary intake such as cooking skills, types of shops used and cooking facilities as well as household structure, employment, education, smoking, drinking and physical activity; dietary data collection on four non-consecutive days using a multiple-pass $24 \mathrm{~h}$ recall method; physical measurements, including height, weight and blood pressure; a blood sample to measure nutritional status indices (age $\geq 8$ years only)

The survey achieved a final response rate of eligible individuals classified as fully productive (i.e. completing three or four dietary recalls) of $55 \%$. A total of 3728 individuals of all ages ( $\geq 2$ years old), drawn from a total of 2477 households were included. Blood samples were obtained from about half the fully-productive respondents.

LIDNS was funded by the Food Standards Agency and conducted by the National Centre for Social Research with King's College London and University College London. 